\title{
Atomic diffusion in star models of type earlier than $G$
}

\author{
P. Morel and F. Thévenin \\ Département Cassini, UMR CNRS 6529, Observatoire de la Côte d’Azur, BP 4229, 06304 Nice Cedex 4, France \\ Received 7 February 2002 / Accepted 15 May 2002

\begin{abstract}
We introduce the mixing resulting from the radiative diffusivity associated with the radiative viscosity in the calculation of stellar evolution models. We find that the radiative diffusivity significantly diminishes the efficiency of the gravitational settling in the external layers of stellar models corresponding to types earlier than $\approx \mathrm{G}$. The surface abundances of chemical species predicted by the models are successfully compared with the abundances determined in members of the Hyades open cluster. Our modeling depends on an efficiency parameter, which is evaluated to a value close to unity, that we calibrate in this study.
\end{abstract}

Key words. diffusion - stars: abundances - stars: evolution - Hertzsprung-Russell (HR) and C-M diagrams

\section{Introduction}

Microscopic diffusion, sometimes named "atomic" or "element" diffusion, when used in the computation of models of main sequence stars produces (see Chaboyer et al. 2001 for an abridged revue) a change in the surface abundances from their primordial values and a shift of the evolutionary tracks towards lower luminosities and temperatures (Morel \& Baglin 1999), consequently increasing the expected age of binaries and clusters. For the Sun, the insertion of microscopic diffusion has spectacularly improved the agreement between the theoretical and the "seismic" model (e.g. Christensen-Dalsgaard et al. 1996). Indeed, for low mass stars and for population II stars, atomic diffusion eliminates, at least partially, discrepancies between observations and theoretical models (Morel \& Baglin 1999; Lebreton et al. 1999; Cayrel et al. 1999; Chaboyer et al. 2001; Salaris \& Weiss 2001).

For stellar models with mass larger than $\approx 1.4 M_{\odot}$, the use of microscopic diffusion alone produces at the surface an important depletion of helium and heavy elements and a concomitant enhancement of the hydrogen content. As an example, for a $2 M_{\odot}$ model computed with atomic diffusion, at the age of $20 \mathrm{Myr}$ the surface mass fraction of hydrogen increased from $X=0.70$ to $X \gtrsim 0.98$, while helium and heavy elements are strongly depleted. This strong depletion, which increases with the mass of the star, is not observed either by Varenne \& Monier (1999) in stars belonging to the Hyades open cluster or in OB associations (Daflon et al. 2000) and in the Orion association (Cunha \& Lambert 1994).

There is no possibility of observing helium in main sequence stars of intermediate mass to check its depletion; such

Send offprint requests to: P. Morel, e-mail: Pierre.Morel@obs-nice.fr stars do not show low surface metallicities. This large helium depletion is not likely to be observed in main sequence B-stars, as supported by their spectral classification which is mainly based on the relative strength of the helium spectral lines.

The large efficiency of the atomic diffusion in the outer layers results from the large temperature and pressure gradients. For models of stars with types later than $\approx$ G, i.e. $M_{\star} \lesssim 1.2 M_{\odot}$, the external convection zone acts as a "reservoir" which inhibits atomic diffusion. On the contrary, for stars with masses larger than $\gtrsim 1.4 M_{\odot}$, the external helium and hydrogen convection zones are so tiny that the reservoir is not large enough to be efficient.

Such misleading physics in the calculation of microscopic diffusion coefficients (e.g. Cox et al. 1989; Proffitt \& Michaud 1991; Michaud \& Proffit 1993; Thoul et al. 1994) are very unlikely owing to the high degree of sophistication of the method based on the kinematical theory of gases (Burgers 1969) and also as seen by its success when applied to the Sun. Clearly, a physical process which inhibits the efficiency of microscopic diffusion is lacking.

Among physical processes acting against gravity, recent developments were focused on:

- The radiative accelerations, which are efficient only in the case of chemical species with a large charge, e.g. iron. As such "metals" have small relative abundances, the radiative accelerations are not efficient limiting the sedimentation of helium and heavy elements as a whole (e.g. Alecian et al. 1989; Turcotte et al. 1998a).

- The turbulent mixing generated by the shear created by the differential rotation between the solar convection zone and the radiative core (Richard et al. 1996; Gabriel 1997; Brun et al. 1998). It has been recently claimed (Schatzman et al. 2000) that the shear instability invoked to produce this 
turbulent mixing only concerns a narrow region and may be not as efficient as expected.

- The rotation-induced mixing accounting for the transport of angular momentum explains well the hot side of the $\mathrm{Li}$ dip in the Hyades (Talon \& Charbonnel 1998).

- The use of a hypothetical mixed reservoir with an ad-hoc adjusted size (e.g. Turcotte et al. 1998b).

- The refinement of the physical description. In the calculations of $\mathrm{G}$ to A type star models (Turcotte et al. 1998a, 1998b; Richer et al. 2000), the local mean Rosseland opacity is updated at each time step, according to local changes in the density and temperature, but also in the genuine mass fractions of chemical species. In some favorable situations the authors have obtained the formation of an "iron" mixed convective zone that inhibits the efficiency of the atomic diffusion. Even with this noticeable improvement of the modeling, several difficulties remain; in particular the models do not reproduce the abundances of star members of the Hyades open cluster as observed by Varenne \& Monier (1999); their Figs. 5 to 8 for elements from $\mathrm{C}$ to $\mathrm{Ba}$ only reveal a mild depletion by a factor $\approx 3$ for $6600 \mathrm{~K} \leq$ $T_{\text {eff }} \leq 7800 \mathrm{~K}$. Also the theoretical strong depletions predicted by Turcotte et al. (1998b) for effective temperatures larger than $6600 \mathrm{~K}$ are not observed.

- The influence of the stellar mass-loss described phenomenologically (Chaboyer et al. 1999) in the modeling of Procyon A.

In this exploratory work, we focus on the role of the radiative diffusivity generated by the photon-ion collisions that is not presently taken into account in the microscopic diffusion coefficients. As we shall see, the photon-ion collisions are an efficient physical process that inhibits the large sedimentation of helium and heavy elements in outer layers of main sequence star models with types earlier than $\approx \mathrm{G}$.

In Sect. 2 we present a simplified phenomenological model of the radiative diffusivity resulting from photon-ion collisions. Section 3 briefly describes the theoretical framework used for constructing stellar evolution models. In Sect. 4 we estimate the radiative diffusivity parameter connecting the radiative kinetic viscosity to the radiative diffusivity; our investigation is based mainly on observed abundances of $\mathrm{C}, \mathrm{O}, \mathrm{Fe}$ and $\mathrm{Mg}$ in stars belonging to the Hyades open cluster as reported by Varenne \& Monier (1999). Illustrations are given in Sect. 5 and, in Sect. 6, we summarize and discuss the main results of the present investigation.

\section{Radiative diffusivity}

Apparently, the radiative diffusivity, first used by Baglin (1972), has not been introduced for intermediate stellar mass models, although implemented in the evolution of models of larger mass (Heger et al. 2000). According to the standard description of the micro-physics of gases (e.g. Burgers 1969; Mihalas \& Weibel-Mihalas 1984, Chap. 1) the viscous stresses due to electric forces tie up the constituent particles, electrons

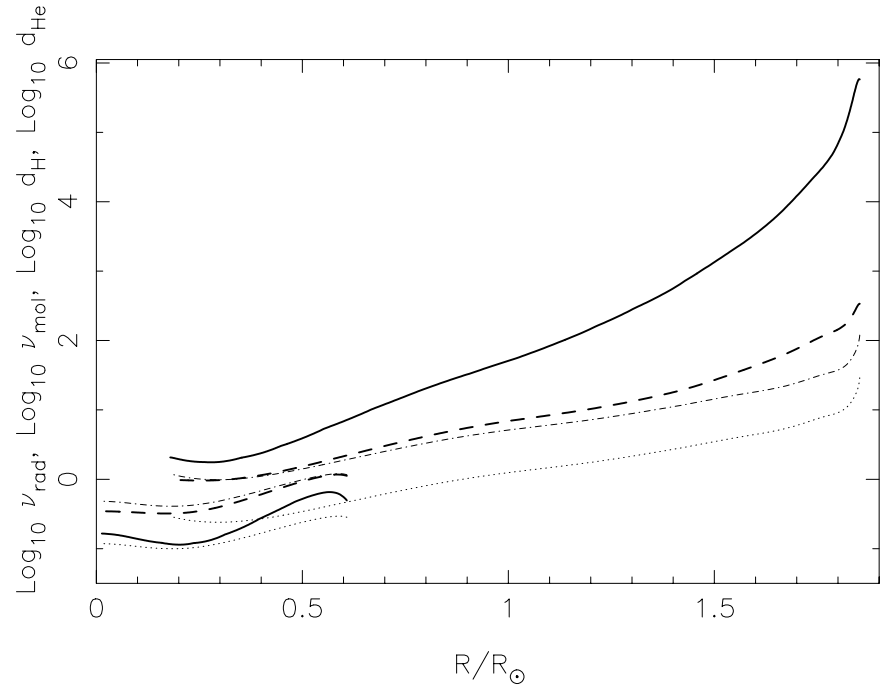

Fig. 1. Profiles of kinetic radiative viscosity $v_{\text {rad }}$ (heavy full), kinetic molecular viscosity $v_{\text {mol }}$ (heavy dashed), hydrogen diffusivity $d_{\mathrm{H}}$ (dotdash-dot-dash) and helium diffusivity $d_{\mathrm{He}}$ (dotted) with respect to radius in the radiative zones of a $1 M_{\odot}\left(R \leq 0.61 R_{\odot}\right)$ and in a $1.8 M_{\odot}$ stellar models at age $700 \mathrm{Myr}$. The kinematic molecular and radiative viscosities and the diffusivities are in c.g.s. $\left(\mathrm{cm}^{2} \mathrm{~s}^{-1}\right)$ units.

and ions. In an astrophysical plasma with radiation, the kinematic viscosity $v$ has two components:

$v=v_{\mathrm{mol}}+v_{\mathrm{rad}}$.

The kinematic molecular viscosity, $v_{\text {mol }}$, results from energy exchanges between thermal collisions leading to excitation and ionization of atoms and ions. Thus, the thermal collisions generate a "molecular mixing" resulting from the molecular diffusivity associated with $v_{\text {mol }}$. The kinematic molecular viscosity is approximated by (Schatzman 1977):

$v_{\mathrm{mol}} \simeq 2.21 \times 10^{-15} \frac{T^{\frac{5}{2}}}{\rho} \frac{1+7 X}{3 \ln \Lambda}$,

for a mixture of hydrogen and helium, assuming that the heavy elements have a negligible effect. $T$ is the temperature, $\rho$ is the density, $X$ is the hydrogen mass fraction and $\ln \Lambda$ is the socalled Coulomb logarithm (e.g. Michaud \& Proffit 1993). With the more elaborate description based on the kinetic theory of gases (Burgers 1969), the molecular diffusion has two components: the isotropic one, being the molecular diffusivity that generates a mixing and the anisotropic one, responsible for the gravitational sedimentation in stars.

The kinematic radiative viscosity $v_{\text {rad }}$ arises because photons deposit their momentum in the fluid element into which they are absorbed. Thus, the radiative collisions generate a "radiative mixing" resulting from the radiative diffusivity associated with $v_{\text {rad }}$. In the optically thick medium the kinematic radiative viscosity is expressed as (Thomas 1930; Mihalas \& Weibel-Mihalas 1984, p. 461-472):

$v_{\mathrm{rad}} \equiv \frac{4}{15} \frac{a T^{4}}{c \kappa \rho^{2}}$

where $a$ is the radiation density constant, $c$ is the speed of light in vacuum and $\kappa$ is the mean Rosseland opacity. 
As already emphasized by several authors more than seventy years ago (e.g. Milne 1930), the radiative viscosity is large in the external layers of stars (Baglin 1972). Figure 1 shows the profiles, with respect to radius, of $v_{\mathrm{mol}}$ and $v_{\mathrm{rad}}$ in the radiative zones of two main sequence stellar models of respectively $1.0 M_{\odot}$ and $1.8 M_{\odot}$ at age $700 \mathrm{My}$. In the radiative zone of the $1 M_{\odot}$ model $\left(R \leq 0.61 R_{\odot}\right)$, the total kinematic viscosity mainly results from the kinematic molecular viscosity while in the radiative outermost layers of the $1.8 M_{\odot}$ model, the kinematic radiative viscosity overcomes the kinematic molecular viscosity by several orders of magnitude. The large value of the radiative viscosity in outer layers of stars is associated with a large radiative diffusivity that makes a significant contribution to the mixing. Like the diffusion due to molecular collisions, the radiative diffusion has an isotropic component, the radiative diffusivity generating mixing, and an anisotropic component causing the radiative accelerations that act against gravity (Alecian 1996).

For stars with mass $M_{\star} \lesssim 1.2 M_{\odot}$ the effect of radiative diffusion is swallowed up by the mixing in the external convection zone, as in the Sun. For stars of types earlier than G, with increasing mass (i.e. effective temperature) the external convective zones become smaller and, in outer layers, the radiative diffusivity is a process which acts more and more efficiently against the gravitational sedimentation.

In the most elaborated stellar evolution codes, the coefficients of microscopic diffusion are derived using the Burgers (1969) formalism (e.g. Cox et al. 1989; Proffitt \& Michaud 1991; Michaud \& Proffit 1993; Thoul et al. 1994). But, in the standard framework of the calculation of collision integrals and resistance coefficients (Burgers 1969; Paquette et al. 1986), only the ion-ion and electron-ion collisions are taken into account and the derived diffusivity corresponds only to $v_{\mathrm{mol}}$.

Figure 1 also shows the profiles of the hydrogen $d_{\mathrm{H}}$ and helium $d_{\mathrm{He}}$ molecular diffusivities. Note that $d_{\mathrm{H}}, d_{\mathrm{He}}$ and $v_{\mathrm{mol}}$ have similar profiles. This behavior suggests a linear relationship, with a dimensionless coefficient close to unity between the molecular diffusivity and the kinetic molecular viscosity.

It is out of the scope of this exploratory work to compute the diffusion coefficients including both the thermal and the radiative collisions according to the Burgers' (1969, Sect. 18 and 60) formalism. We express the diffusivity associated with $v_{\text {rad }}$ by a diffusion coefficient proportional to the kinematic radiative viscosity:

$d_{\mathrm{rad}} \equiv D_{\mathrm{R}} v_{\mathrm{rad}}$

The efficiency factor, $D_{\mathrm{R}}$, is a dimensionless phenomenological parameter to be calibrated. Hereafter, we shall refer to $D_{\mathrm{R}}$ as the "radiative diffusivity parameter". We assume that $D_{\mathrm{R}}$ is constant even if, a priori, there is no reason for a unique value to apply for all atoms and ions of all chemical species and in all stellar conditions.

To illustrate the effect of the radiative diffusivity, using the results of Sect. 5, Fig. 2 shows the abundance profiles with respect to radius in a $1.8 M_{\odot}$ main sequence stellar model at age $570 \mathrm{Myr}$ computed respectively with $\left(D_{\mathrm{R}}=1.0\right)$ and without $\left(D_{\mathrm{R}}=0.0\right)$ radiative diffusivity. Note the small surface abundances of metals predicted by the model computed without radiative diffusivity and the realistic values obtained with $D_{\mathrm{R}}=1.0$.

The simplified description of the radiative diffusivity introduced in this paper is phenomenological and preliminary. Particularly it ignores the ionization stage of elements which could modify the efficiency of the radiative diffusivity of chemical species under consideration. It obviously needs further extended development based on the kinetic theory of gases with radiation.

\section{Computation of models and physical inputs}

Stellar modeling: Stellar models have been computed using the cEsam code (Morel 1997). The physics employed is the same as in Morel et al. (2000). The stellar evolution sequences are initialized with homogeneous pre-main sequence models. We follow in detail the abundances of all chemical species of our diffusion network. The set of evolutionary differential equations fulfilled by the abundances of the isotopes is numerically integrated using a finite element method ${ }^{1}$. The microscopic diffusion coefficients are derived from the set of Burgers' (1969, formula 18.1-18.2) flow equations. The charge of a given isotope is taken as the average ${ }^{2}$ charge over all its ionization states. The collision integrals and resistance coefficients are taken from the tabulations of Paquette et al. (1986). For each ion, the coefficient of radiative diffusivity is added to the coefficient of molecular diffusivity.

Input physics: The EFF equation of state (Eggleton et al. 1973) was used, together with opacities determined by Rogers et al. (1996) using the solar mixture of Grevesse \& Noels (1993), complemented at low temperatures by Alexander \& Ferguson (1994) opacities. In the convection zones the mixing is insured by a large turbulent diffusion coefficient and the temperature gradient is computed according to the Böhm-Vitense (1958) convection theory; the mixing-length parameter is fixed to the value in the standard solar model computed with the same physics ${ }^{3}$. The atmosphere is restored using Hopf's atmospheric law (Mihalas 1978). The element diffusion is not computed in the atmosphere where the chemical composition is fixed at the value in the outermost shell of the envelope.

Nuclear network: The relevant nuclear reaction rates are taken from the NACRE compilation (Angulo et al. 1999). The nuclear network contains the isotopes: ${ }^{1} \mathrm{H},{ }^{2} \mathrm{H},{ }^{3} \mathrm{He},{ }^{4} \mathrm{He},{ }^{7} \mathrm{Li},{ }^{7} \mathrm{Be},{ }^{12} \mathrm{C}$, ${ }^{13} \mathrm{C},{ }^{14} \mathrm{~N},{ }^{15} \mathrm{~N},{ }^{16} \mathrm{O}$ and ${ }^{17} \mathrm{O}$. They are processed by the $\mathrm{PP}+\mathrm{CNO}$ thermonuclear reactions relevant for the main sequence evolution. The elements ${ }^{2} \mathrm{H}$ and ${ }^{7} \mathrm{Be}$ are set at equilibrium.

Diffusion network: Along the main sequence the chemical species, heavier than ${ }^{17} \mathrm{O}$, are not nuclearly processed.

\footnotetext{
1 The derivation of the equations and the algorithms are detailed in the "Notice de CESAM" and available on request on the WEB site http://www . obs-nice. fr/morel/CESAM. html.

2 Weighted by the ionization rates.

3 But the inefficient radiative diffusivity, see Sect. 4.
} 

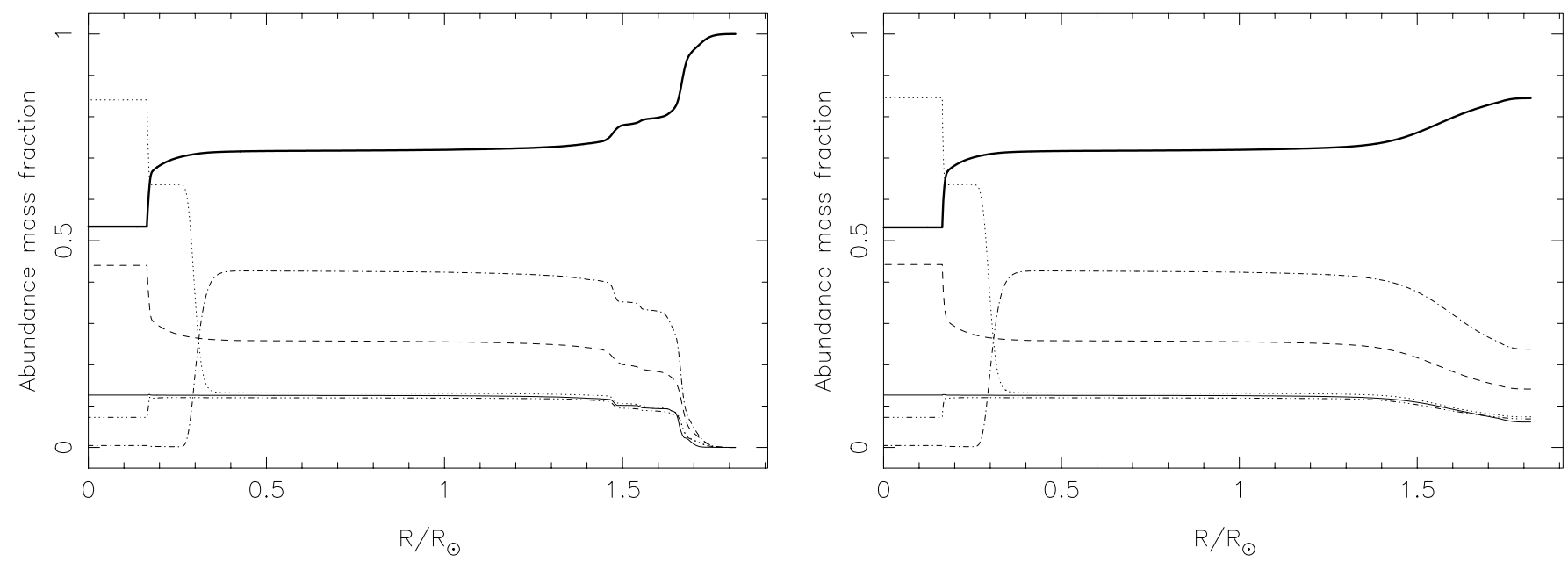

Fig. 2. Profiles, with respect to radius, of abundance mass fractions in a $1.8 M_{\odot}$ "Hyades" stellar model at age 570 Myr computed with $D_{\mathrm{R}}=0$ (left) and $D_{\mathrm{R}}=1$ (right). $\mathrm{H} \times 1$ (heavy full), $\mathrm{He} \times 1$ (dashed), $\mathrm{C} \times 100$ (dot-dash-dot-dash), $\mathrm{N} \times 100$ (dotted), $\mathrm{O} \times 100$ (dash-dot-dot-dot-dash), $\mathrm{Fe} \times 100$ (thin full).

However, their abundances vary with respect to time owing to diffusion processes, contributing to changes of $Z$, thus generating opacity variations. Morel et al. (1997) introduced a dummy "mean metal" to approximate the mean opacity changes caused by the variations of $Z$ resulting from microscopic diffusion. To mimic more closely the effects of low and high charged chemical species, we use here two dummy mean metals. The first simulates the metals heavier than potassium. For the solar mixture of Grevesse \& Noels (1993) used so far, their mean ${ }^{4}$ charge and mean atomic weight are respectively 26 and 55 . Therefore, a dummy "iron", labeled hereafter $\left\{{ }^{55} \mathrm{Fe}\right\}$, is well representative of the heaviest chemical species. Indeed, the second dummy metal simulates the isotopes with atomic numbers from 9 to 20 , and also complements the mixture. Its charge and atomic mass are respectively 12 and 24, corresponding to a "dummy" magnesium $\left\{{ }^{24} \mathrm{Mg}\right\}$. For the sake of simplicity we shall approximate $\left\{{ }^{55} \mathrm{Fe}\right\}$ and $\left\{{ }^{24} \mathrm{Mg}\right\}$ with respectively the iron and the magnesium abundances measured spectroscopically.

Initial abundances: To fulfill the basic relationship $X_{\text {ini }}+Y_{\text {ini }}+$ $Z_{\text {ini }} \equiv 1$, the initial abundances of isotopes are derived (1) from the isotopic ratios of nuclides (Anders \& Grevesse 1989), (2) from the initial mass fraction of helium, $Y_{\text {ini }} \equiv{ }^{3} \mathrm{He}_{\text {ini }}+{ }^{4} \mathrm{He}_{\text {ini }}$, and (3) from the initial mass fraction of heavy elements relative to hydrogen $\left(\frac{Z}{X}\right)_{\text {ini }}$. As deuteron is assumed at equilibrium the initial amount of ${ }^{3} \mathrm{He}$ is enhanced by the initial meteoritic amount of deuteron (Geiss \& Reeves 1972).

Failing anything better, we use the meteoritic values of Anders \& Grevesse (1989) for the initial fractions, by number, of metals within $Z:{ }^{12} \mathrm{C}+{ }^{13} \mathrm{C}=0.2455,{ }^{14} \mathrm{~N}+{ }^{15} \mathrm{~N}=$ $0.06458,{ }^{16} \mathrm{O}+{ }^{17} \mathrm{O}=0.5130$. From these data one infers the initial abundances for the dummy metals $\left\{{ }^{24} \mathrm{Mg}\right\}=0.1631$, $\left\{{ }^{55} \mathrm{Fe}\right\}=0.01563$. For the initial isotopic ratio (by number) we use ${ }^{3} \mathrm{He} /{ }^{4} \mathrm{He}=1.1 \times 10^{-4}$ (Gautier \& Morel 1997).

\footnotetext{
${ }^{4}$ Weighted by number.
}

For any chemical species $\mathcal{X}$ we shall refer to $\left[\frac{X}{\mathrm{H}}\right]$ as the quantity:

$$
\left[\frac{X}{\mathrm{H}}\right] \equiv \log \left(\frac{\mathcal{X}}{\mathrm{H}}\right)_{t}-\log \left(\frac{\mathcal{X}}{\mathrm{H}}\right)_{t=0}
$$

which is a measurement of the depletion of $\mathcal{X}$ at the age $t$ under consideration.

\section{Constrains on the radiative diffusivity parameter}

The radiative diffusivity parameter $D_{\mathrm{R}}$ is estimated by comparing relevant observations to theoretical results. Firstly, owing to the high accuracy of helioseismological observations, some insights can be gained using the standard solar model despite the inhibition, by the convective mixing, of the microscopic diffusion in the outer layers. Secondly, the most sensitive observations, relevant to the atomic diffusion, are the surface abundances of chemical species of members of well-observed open clusters. When the age of the cluster is not older than $\approx 1 \mathrm{Gyr}$, the effects of element diffusion remain small for $\approx \mathrm{G}$ dwarfs and the initial abundances can be reasonably inferred. For intermediate mass stars we shall estimate the value of the radiative diffusivity parameter using the observational material of the Hyades open cluster and, for early type stars, we shall use observations of Orion association.

Solar models: In the external layers of a solar model, the radiative diffusivity is swallowed up by the convective mixing. Beneath the convection zone, where precise informations on the internal structure result from helioseismology, the effects of radiative diffusivity should be compatible with the relevant properties of the nowadays very precise standard solar model. This assertion leads to an estimate of an upper limit for $D_{\mathrm{R}}$.

We have computed calibrated solar models for the following values of the radiative diffusion parameter $D_{\mathrm{R}}=0,1,5$, 
Table 1. Depth of the convection zone (solar unit), surface isotopic ratio of ${ }^{3} \mathrm{He}$ versus ${ }^{4} \mathrm{He}$, surface helium mass fraction $Y_{\mathrm{s}}$, small difference $\delta v_{02}(\mu \mathrm{Hz})$ and $[\mathrm{Li}]_{\mathrm{s}}$ lithium abundance in dex $(H \equiv 12)$ in calibrated solar models computed with different values for the radiative diffusivity parameter $D_{\mathrm{R}}$.

\begin{tabular}{llllll}
\hline \hline$D_{\mathrm{R}}$ & $R_{\mathrm{zc}}$ & {$\left[\frac{{ }^{3} \mathrm{He}}{{ }^{4} \mathrm{He}}\right]_{\mathrm{s}}$} & $Y_{\mathrm{s}}$ & $\delta v_{02}$ & {$[\mathrm{Li}]_{\mathrm{s}}$} \\
\hline 0 & 0.7127 & 4.343 & 0.2430 & 9.09 & 2.603 \\
1 & 0.7128 & 4.345 & 0.2430 & 9.10 & 2.609 \\
5 & 0.7129 & 4.353 & 0.2431 & 9.12 & 2.608 \\
10 & 0.7131 & 4.359 & 0.2433 & 9.15 & 2.607 \\
50 & 0.7142 & 4.381 & 0.2448 & 9.32 & 2.502 \\
\hline
\end{tabular}

Observations

$R_{\mathrm{zc}}=0.713 \pm 0.001$

$\left[\frac{{ }^{3} \mathrm{He}}{4}\right]_{\mathrm{S}}=4.4 \pm 0.4$

$\mathrm{Y}_{\mathrm{s}}=0.240 \pm 0.009$

$\delta v_{02}=9.01 \pm 0.10$

\section{References}

Basu \& Antia (1995)

Bodmer et al. (1995)

Basu (1997)

Grec et al. (1997).

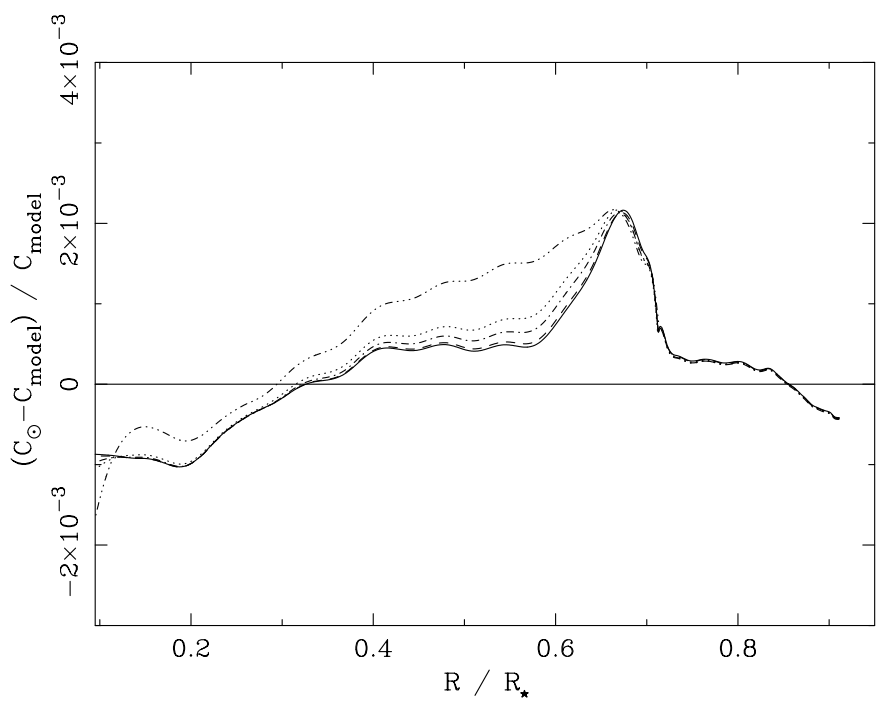

Fig. 3. Relative differences in sound velocities between the Sun and calibrated solar models with $D_{\mathrm{R}}=0,1,5,10,50$ respectively: full, dashed, dot-dash-dot-dash, dotted, dash-dot-dot-dot-dash.

10, 50. To compare solar models with helioseismological observations, we use improved physical data and standard solar initial chemical composition (details can be found in Morel et al. 1997). In the range $0.1 R_{\odot} \lesssim R \lesssim 0.9 R_{\odot}$ where the inversions of the helioseismic data are reliable, Fig. 3 shows the relative differences between the sound speed in the calibrated solar models and the seismic sound speed "experimental" results of Turck-Chièze et al. (1997). For the models computed with $D_{\mathrm{R}} \lesssim 10$, the relative discrepancy between the sound speed in the Sun and in the model are not larger than a few $10^{-3}$. Table 1 shows that the theoretical values of standard solar characteristics are all compatible with observations; nonetheless, these data are not strongly dependant on $D_{\mathrm{R}}$.

From Fig. 3, we estimate that a reasonable upper limit for the radiative diffusivity parameter is close to $\left\lceil D_{\mathrm{R}}\right\rceil \lesssim 10$.

Massive stars: Massive stars, belonging to OB associations, provide a test of efficiency for the radiative diffusivity. For stars having masses from 7 to $17 M_{\odot}$ observations do not reveal any chemical abundances depletion with respect to the effective temperature, i.e. masses, neither for $\mathrm{C}, \mathrm{N}$ and $\mathrm{O}$ nor for heavier elements, as it is shown in Fig. 3 of Daflon et al. (2000) or in Fig. 17 of Cunha \& Lambert (1994). These recent star-forming regions are younger than $20 \mathrm{Myr}$ and most of the stars analysed in Cunha \& Lambert (1994) have an age supposed to be around $7 \mathrm{Myr}$.

In models of such massive stars including element diffusion alone $\left(D_{\mathrm{R}}=0\right)$, helium and metals empty the outer layers in an evolutionary time shorter than $\approx 10 \mathrm{Myr}$. In these models the outer hydrogen and helium convection zones are so tiny that the convective mixing is ineffective. Resulting from the large pressure and temperature gradients, the element diffusion is very efficient, leading to a large sedimentation of helium and heavy elements. The inclusion of the radiative diffusivity generates a mixing which avoids this unrealistic result. Due to the large effective temperature $\left(T_{\text {eff }} \approx 10000 \mathrm{~K}\right)$, the kinematic radiative viscosity is large in the outer layers, see Eq. (2), and so the radiative diffusivity; that generates an efficient mixing inhibiting the large efficiency of the element diffusion and allows one to reproduce the observed absence of depletion of helium and metals.

To estimate $D_{\mathrm{M}}$, adopting a mean age of $7 \mathrm{Myr}$, we have computed the evolved abundances of $\mathrm{C}, \mathrm{N}, \mathrm{O}$ and $\mathrm{Fe}$ for stellar masses from 7 to $17 M_{\odot}$ for different values of the radiative diffusivity parameter. In these early type star models the radiative diffusivity inhibits the efficiency of the element diffusion as soon as $D_{\mathrm{R}}$ is larger than the lower limit $\left\lfloor D_{\mathrm{R}}\right\rfloor=0.05$.

Hyades: The Hyades cluster is a nice laboratory for testing stellar structure and evolution theory because of its numerous well analysed stars and its well measured distance. We have computed star models taking into account the atomic diffusion including radiative diffusivity for different values of $D_{\mathrm{R}}$. We refer to Lebreton et al. (2001) for the cluster parameters. We adopt a metallicity of $\left[\frac{\mathrm{Fe}}{\mathrm{H}}\right]=+0.14 \pm 0.03 \mathrm{dex}$ and an age of $550 \pm 50 \mathrm{Myr}$ as our models are computed without overshoot of the convective core. Alike for the Sun, a typical G dwarf Hyades member of $1.1 M_{\odot}$ is not affected by the radiative diffusivity but, owing to the element diffusion, the initial helium mass fraction needs to be enhanced up to $Y_{\text {ini }}=0.259$ to ends at $Y=0.255$ after $550 \mathrm{Myr}$ of evolution. Also, the initial metallicity needs to be $[\mathrm{Fe} / \mathrm{H}]_{\text {ini }}=0.147 \mathrm{dex}$ to reach $[\mathrm{Fe} / \mathrm{H}]=0.140 \mathrm{dex}$ at the age of Hyades. For our "Hyades" models we use the following initial mass fractions (in dex with respect to $\mathrm{H} \equiv 12):{ }^{3} \mathrm{He}=8.056,{ }^{4} \mathrm{He}=11.56,{ }^{12} \mathrm{C}=9.738$, ${ }^{13} \mathrm{C}=7.818,{ }^{14} \mathrm{~N}=9.299,{ }^{15} \mathrm{~N}=6.896,{ }^{16} \mathrm{O}=10.24,{ }^{17} \mathrm{O}=$ $6.847,\left\{{ }^{24} \mathrm{Mg}\right\}=9.416,\left\{{ }^{55} \mathrm{Fe}\right\}=9.838$.

Figure 4 shows the surface abundances of $\mathrm{C}, \mathrm{O}, \mathrm{Mg}$, and $\mathrm{Fe}$ with respect to effective temperature in models computed with $D_{\mathrm{R}}=0.0,0.5,1.0,5.0,9.0$, together with the observations of Varenne \& Monier (1999). For main sequence stars, with effective temperatures between $6600 \mathrm{~K} \leq T_{\text {eff }} \leq 7800 \mathrm{~K}$, the depletions of abundances are decreasing with respect to the radiative diffusivity parameter. With $D_{\mathrm{R}} \cong 1$ the depletions amount to about a factor $\approx 2-3$ for all chemical species; that corresponds 

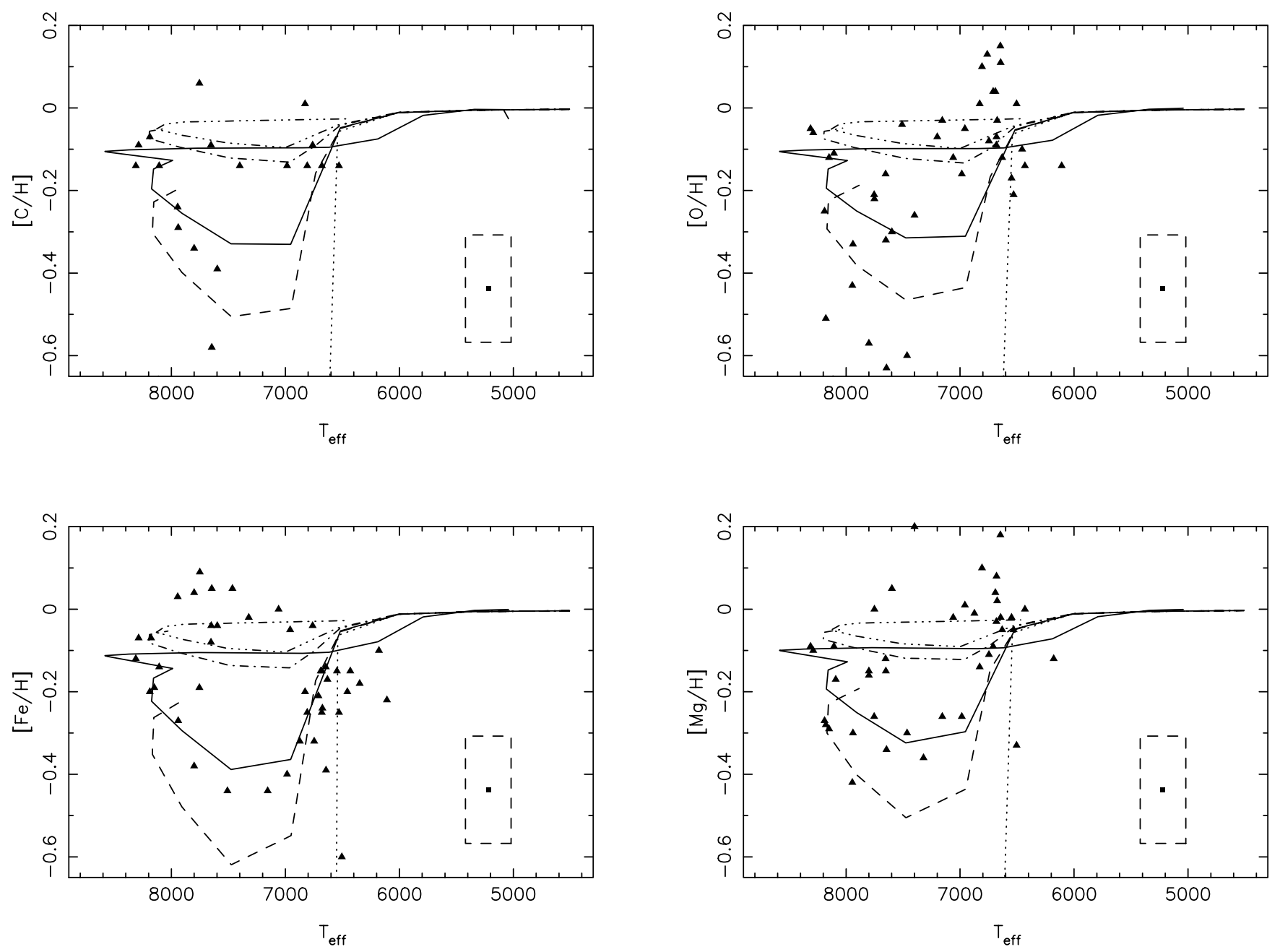

Fig. 4. Surface abundances (in dex) with respect to effective temperature of $\mathrm{C}, \mathrm{O}, \mathrm{Fe}$ and $\mathrm{Mg}$ for a sample of "Hyades" stellar models at the age of $570 \mathrm{My}$ computed with $D_{\mathrm{R}}=0.0$ (dotted), $D_{\mathrm{R}}=0.5$ (dashed), $D_{\mathrm{R}}=1.0$ (full), $D_{\mathrm{R}}=5.0$ (dash-dot-dash-dot) and $D_{\mathrm{R}}=9.0($ dashdot-dot-dot-dash). The triangles correspond to the observations of Varenne \& Monier (1999) corrected from the metallicity of Hyades. Dashed rectangles delimit the uncertainty domains of observations.

to the observations even if, for oxygen, the distribution of the observed abundances are rather scattered, probably resulting from non-LTE effects or 3D temperature inhomogeneities in the atmosphere (Asplund et al. 2000a).

Taking into account observational error bars, Fig. 4 shows that the best agreement is obtained for $D_{\mathrm{R}} \cong 1$.

\section{Comments on stellar models with $D_{R}=1$}

Using stellar models computed with element diffusion in the following we give two illustrations of the use of radiative diffusivity (1) to estimate the age of Hyades and (2) to give properties of a sample of Pop I stellar models.

Age and predicted abundances of the Hyades stars: So far we have used for the Hyades open cluster the age and the initial chemical composition derived from the work of Lebreton et al. (2001) that does not take into account the element diffusion. Using stellar models computed with $D_{\mathrm{R}}=1.0$, and the corrected initial helium content and metallicity introduced in Sect. 4 , we have reestimated the age by fitting isochrones to the observations of Balachandran (1995). Figure 6 shows the isochrones.

The inclusion of the element diffusion leads to the age $t_{\text {Hyades }}=570 \pm 15 \mathrm{Myr}$, i.e. an increase of $\approx 3 \%$ with respect to the result of Lebreton et al. (2001). As already emphasized by Lebreton et al. (2001) one can see the sensitivity of the age to the loci of the two components of the $\theta$ Tau binary system with $\theta^{2}$ Tau located right on the turn-off.

Figure 5 reproduces the predictions of surface abundances for helium, lithium and nitrogen with respect to the effective temperature. Below $T_{\text {eff }} \lesssim 6000 \mathrm{~K}$ the external convective zone extends deeply enough to reach the area where lithium is nuclearly destroyed, resulting in a low surface abundance. For $6400 \lesssim T_{\text {eff }} \lesssim 6600 \mathrm{~K}$ the lithium abundance is close to its initial value as for the other elements. Above $\gtrsim 6600 \mathrm{~K}$ and until $\approx 8200 \mathrm{~K}$ the depletion due to the element diffusion is more and more counter balanced by the mixing generated by the increasing radiative diffusivity. For stars above the turn-off, 


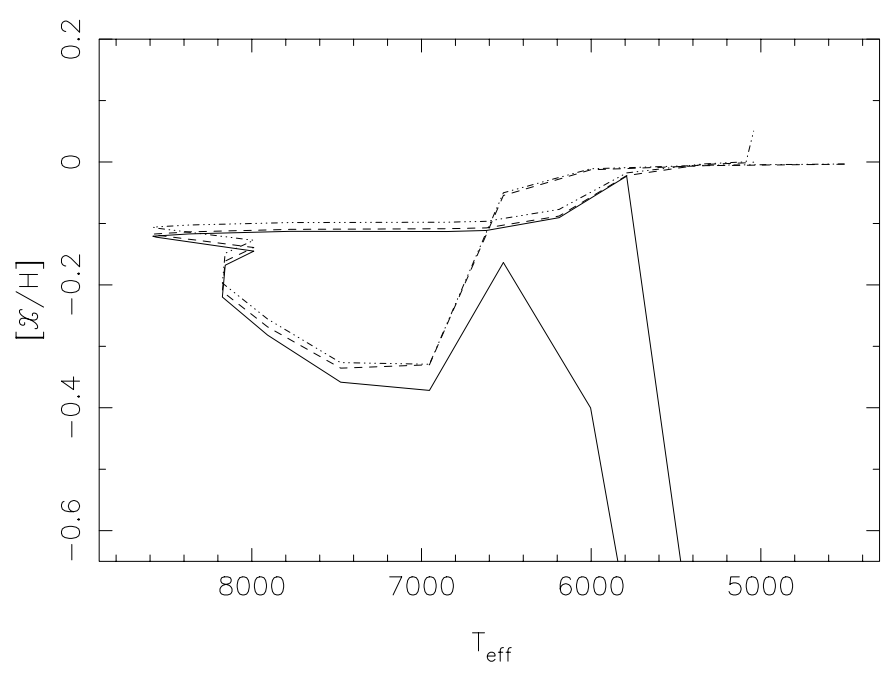

Fig. 5. Profiles of expected surface abundances (in dex) with respect to effective temperature for $\mathcal{X}=\mathrm{He}$ (dashed), $\mathrm{Li}$ (full) and $\mathrm{N}$ (dashdot-dot-dot-dash) for a sample of "Hyades" stellar models at the age of $570 \mathrm{My}$ computed with $D_{\mathrm{R}}=1.0$.

first the evolutionary time scale is small and the element diffusion is less efficient and second, the mixing resulting from the dredge up becomes more and more efficient and the surface abundances keep about their initial values.

Figure 8 shows the surface abundance of lithium with respect to effective temperature. The so-called Li dip (Boesgaard $\&$ Tripicco 1986), located in the vicinity of $T_{\text {eff }} \approx 6500 \mathrm{~K}$ is not reproduced by our models. As in the standard solar model, see Table 1, the radiative diffusivity combined with element diffusion does not suffice to explain the lithium depletion. As clearly demonstrated by Charbonnel et al. (1994) the Li dip does not result from the gravitational settling; according to the present paradigm (Sills \& Deliyannis 2000), it results from the mixing generated beneath the tachocline due to the shear caused by the differences of rotational velocities between the convection zone and the radiative interior (Talon \& Charbonnel 1998). At the age under consideration, according to the rotational status of the star and to the extend of the external convection zone, the mixed region extends towards the part of the envelope where the lithium is nuclearly destroyed. The other elements, except beryllium and boron, being nuclearly destroyed in the core are not affected by this process. The radiative diffusivity then acts together with the other processes to inhibit the gravitational settling, generating the mild depletion of metals as observed by Varenne \& Monier (1999).

For reference, Table 2 shows theoretical surface parameters of our "Hyades" stellar models and reveals significant changes of the surface abundances according to the stellar mass. Therefore, at present, neither the helium mass fraction nor the metallicity have a unique value at the surface of the main sequence star members of the cluster.

A sample of Pop. I stellar models: To investigate the change of the surface abundances resulting from the element diffusion on the main sequence and subgiant stars we have computed typical evolutions of Pop. I stellar models for respectively $1 . M_{\odot}$,

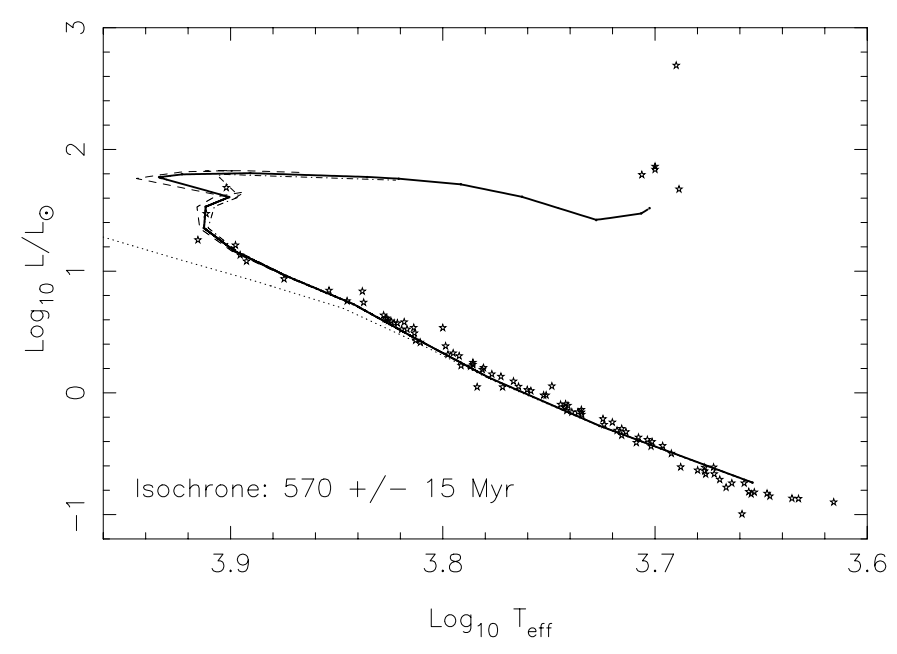

Fig. 6. Isochrones of ZAMS (dotted), $570 \mathrm{Myr}$ (full), $585 \mathrm{Myr}$ (dashdot-dash) and $555 \mathrm{Myr}$ (dashed) for the Hyades open cluster computed with element diffusion and $D_{\mathrm{R}}=1$.

1.4 $M_{\odot}, 1.8 M_{\odot}, 2.5 M_{\odot}$ and 4. $M_{\odot}$. Models are initialized at homogeneous zaMs with solar composition. Figure 7 shows, with respect to age, the profiles of the surface abundance and the corresponding HR diagrams. Along the main sequence, the surface mass fractions of helium and metals are depleted, but along the giant branch, the surface abundances recover about their initial values as a consequence of the dredge-up. Therefore it not possible to derive a unique metallicity for all stars of an open cluster like Hyades.

Note that for the model of $1 M_{\odot}$ at $4.65 \mathrm{Gyr}$, the age of the Sun, we obtain a depletion for iron of $\approx 0.059 \mathrm{dex}$, consistent with the present day adopted difference between photospheric and meteoritic observed abundances (Asplund et al. 2000b).

\section{Discussion}

Radiative diffusivity is a basic physical phenomenon that complements standard element diffusion in stellar modeling. In this exploratory work, we have introduced in the calculation of the microscopic diffusion coefficients, an estimate of the radiative diffusivity resulting from photon-ion collisions that acts predominantly in the external layers of stars of high effective temperature. This simple treatment needs an efficiency factor, the radiative diffusivity parameter $D_{\mathrm{R}}$. We have constrained $D_{\mathrm{R}}$ by the fit of observations chosen independent of rotation. The studies presented in Sect. 4 strongly suggest that a value close to unity, $D_{\mathrm{R}}=1.0_{-0.2}^{+2.0}$, satisfies all the observational constraints. We have adopted a unique value $D_{\mathrm{R}}=1$ for all chemical element species and in all physical conditions.

Without taking into account other physical processes that inhibit the gravitational sedimentation, namely mass-loss, or turbulent mixing generated by rotation or by some other turbulent process, we find that the implementation of the radiative diffusivity inhibits the large gravitational settling resulting from the element diffusion in outer layers of early-type star models with masses larger than $\gtrsim 1.4 M_{\odot}$.

The radiative diffusivity is not able to solve all the open questions of element depletion in stellar models 
Table 2. Typical physical parameters of "Hyades" stellar models computed with $D_{\mathrm{R}}=1$ at age $570 \mathrm{Myr}$. $M_{\star}, T_{\text {eff }}, \log g$ and $M_{\text {bol }}$ are respectively the mass, the effective temperature (in K), the logarithm of the gravity and the bolometric magnitude $\left(M_{\mathrm{bol} \odot}=4.75\right) .\left[\frac{\mathrm{He}}{\mathrm{H}}\right],\left[\frac{\mathrm{C}}{\mathrm{H}}\right],\left[\frac{\mathrm{N}}{\mathrm{H}}\right],\left[\frac{\mathrm{O}}{\mathrm{H}}\right],\left[\frac{\mathrm{Mg}}{\mathrm{H}}\right]$, $\left[\frac{\mathrm{Fe}}{\mathrm{H}}\right]$ are the relative surface abundances with respect to the Hyades one: +0.14 dex.

\begin{tabular}{llllllllll}
\hline \hline$M_{\star} / M_{\odot}$ & $T_{\text {eff }}$ & $\log g$ & $M_{\text {bol }}$ & {$\left[\frac{\mathrm{He}}{\mathrm{H}}\right]$} & {$\left[\frac{\mathrm{C}}{\mathrm{H}}\right]$} & {$\left[\frac{\mathrm{N}}{\mathrm{H}}\right]$} & {$\left[\frac{\mathrm{O}}{\mathrm{H}}\right]$} & {$\left[\frac{\mathrm{Mg}}{\mathrm{H}}\right]$} & {$\left[\frac{\mathrm{Fe}}{\mathrm{H}}\right]$} \\
\hline 0.8 & 4508 & 4.65 & 6.59 & -0.003 & -0.002 & -0.002 & -0.002 & -0.002 & -0.003 \\
1.0 & 5324 & 4.57 & 5.43 & -0.004 & -0.004 & -0.004 & -0.004 & -0.004 & -0.004 \\
1.2 & 6003 & 4.46 & 4.44 & -0.009 & -0.008 & -0.008 & -0.008 & -0.008 & -0.009 \\
1.4 & 6517 & 4.34 & 3.61 & -0.040 & -0.035 & -0.037 & -0.040 & -0.033 & -0.038 \\
1.6 & 6953 & 4.24 & 2.93 & -0.259 & -0.279 & -0.274 & -0.252 & -0.226 & -0.293 \\
1.8 & 7477 & 4.18 & 2.35 & -0.264 & -0.276 & -0.270 & -0.255 & -0.252 & -0.317 \\
2.0 & 7908 & 4.12 & 1.84 & -0.208 & -0.208 & -0.207 & -0.199 & -0.190 & -0.234 \\
2.2 & 8175 & 4.03 & 1.37 & -0.161 & -0.156 & -0.156 & -0.152 & -0.144 & -0.173 \\
2.4 & 8159 & 3.89 & 0.927 & -0.122 & -0.156 & -0.115 & -0.134 & -0.108 & -0.127 \\
2.5 & 7991 & 3.79 & 0.721 & -0.104 & -0.098 & -0.098 & -0.097 & -0.093 & -0.109 \\
2.55 & 8592 & 3.76 & 0.323 & -0.088 & -0.080 & -0.080 & -0.079 & -0.070 & -0.083 \\
2.57 & 7823 & 3.57 & 0.236 & -0.082 & -0.074 & -0.074 & -0.074 & -0.065 & -0.077 \\
2.59 & 5151 & 3.22 & 1.169 & -0.002 & -0.006 & -0.004 & -0.001 & -0.001 & -0.001 \\
\hline
\end{tabular}
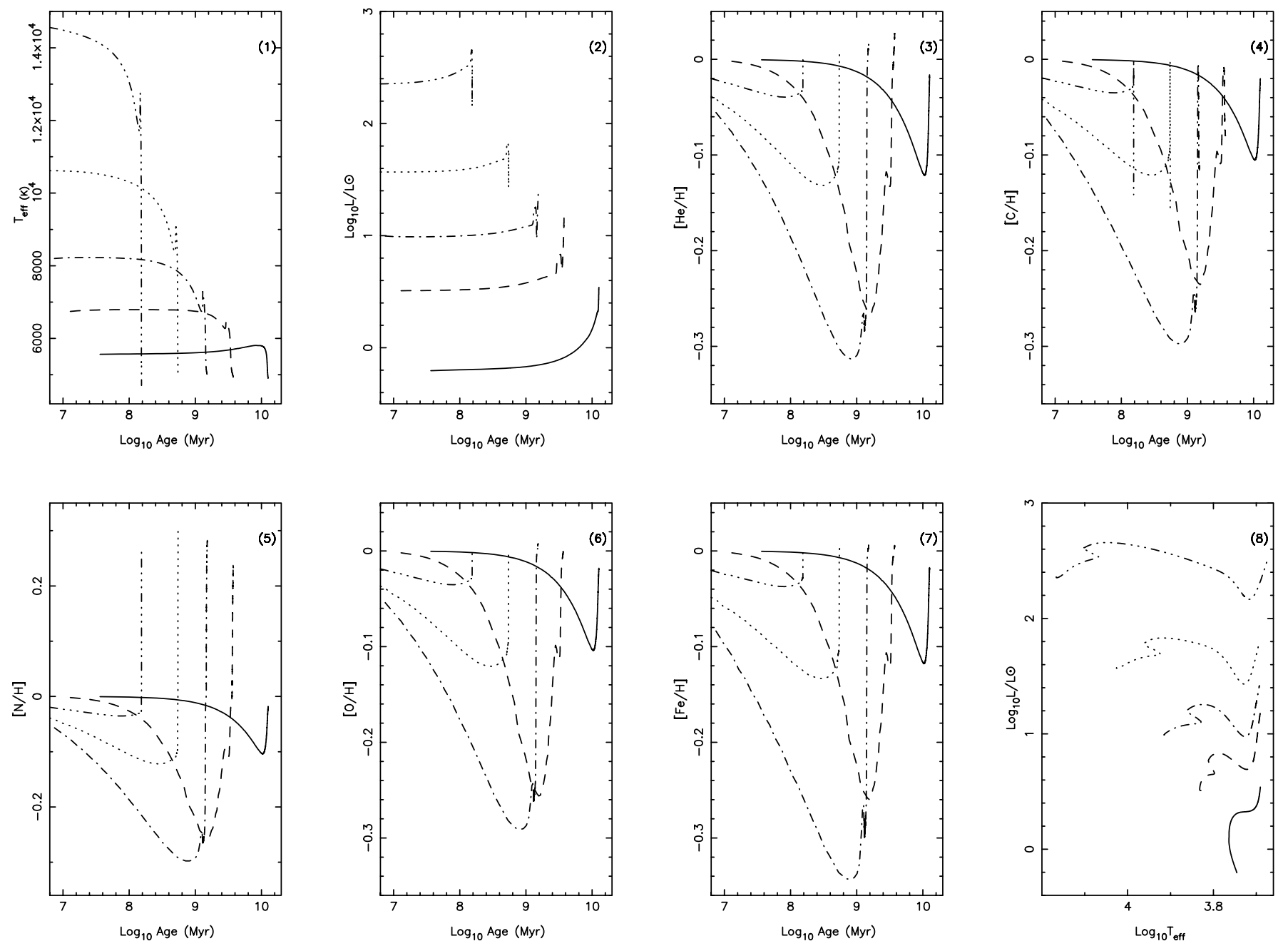

Fig. 7. Panels (1) to (7) show the profiles, with respect to age, of the effective temperature, of the luminosity, of abundance mass fractions at the surface of a sample of Pop. I stellar models computed with $D_{\mathrm{R}}=1.0$. Panel (8) shows the corresponding HR diagram. The labels are self explanatory. The stellar masses are respectively $M_{\star} / M_{\odot}=1.0$ (full), 1.4 (dashed), 1.8 (dot-dash-dot-dash), 2.5 (dotted), 4.0 (dash-dot-dot-dotdash). 


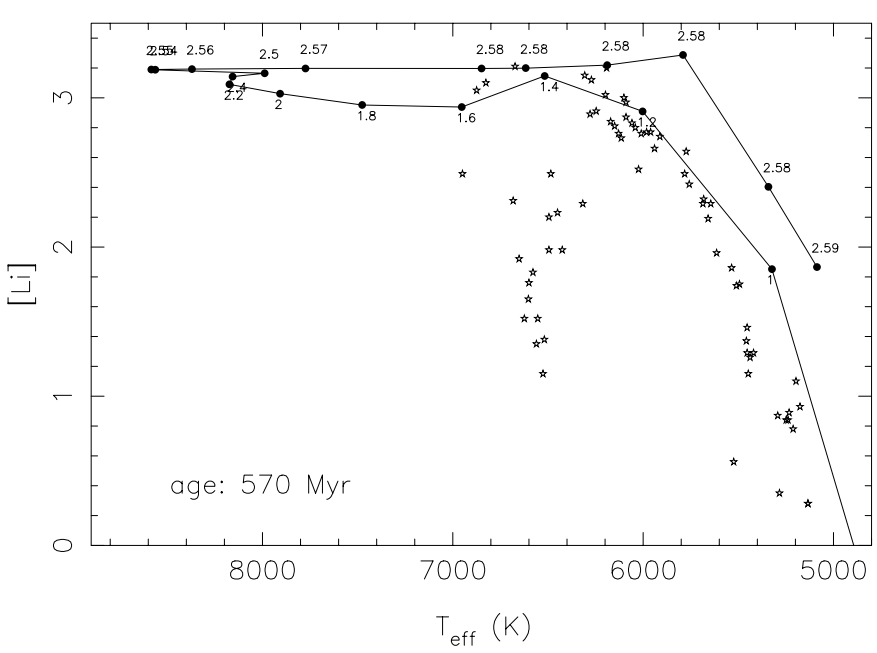

Fig. 8. Surface abundance of lithium with respect to effective temperature of stellar models (full) of the Hyades computed with $D_{\mathrm{R}}=1.0$ at the age of $570 \mathrm{Myr}$. The labels of dots give the mass (solar unit) of models. Observed lithium abundances and effective temperatures of the Hyades field stars $(\star)$ are taken from Balachandran (1995).

(e.g. Balachandran 1995; Sills \& Deliyannis 2000 for reviews). Here, our goal is to emphasize on its large magnitude and to point out that it should be added to the other mixing processes. Radiative diffusivity simply acts together with the mixing induced by rotation (Zahn 1992), the mass-loss (Chaboyer et al. 1999), the turbulent mixing (Schatzman 1969) and the radiative accelerations (Alecian et al. 1989) to inhibit the large element diffusion.

The implementation of the radiative diffusivity in stellar modeling is necessary, in particular, for the calculation of:

- precise models of intermediate mass stars that are targets of asteroseismological experiments like COROT or EDDINGTON,

- theoretical isochrones including element diffusion, for young cluster presenting stars with masses above $1.4 M_{\odot}$ at the turn-off.

Our simplified model takes into account only the mixing generated by the anisotropic component of the radiative diffusion; it ignores the radiative acceleration and so cannot describe the AmFm phenomenon (Michaud et al. 1983; Alecian 1996; Hui-Bon-Hua \& Alecian 1998; Richer et al. 2000).

We are aware that our estimation of the radiative diffusivity is only a first approximation; owing to its efficiency we are confident of the fact that the diffusivity generated by the radiative collisions is a part of the missing physical process responsible for the too large efficiency of the microscopic diffusion coefficient. Our description of the radiative diffusivity is phenomenological and preliminary and needs further extended developments based on the kinetic theory of gases and radiation.

Acknowledgements. We are grateful to A. Baglin for many valuable suggestions and advice concerning the concept of this paper. Fruitful discussions with J.-P. Zahn, Y. Lebreton, G. Alecian, M.-J. Goupil and R. Monier are gratefully acknowledged. We would like to express our thanks to J. Provost for the computation of the small differences of the calibrated solar models. The comments of the anonymous referee significantly improved the presentation of this paper. This research has made use of the Simbad data base, operated at CDS, Strasbourg, France. This work has been performed using the computing facilities provided by the OCA program "Simulations Interactives et Visualisation en Astronomie et Mécanique (SIVAM)".

\section{References}

Alecian, G., Michaud, G., \& Tully, J. 1989, A\&A, 411, 882

Alecian, G. 1996, A\&A, 310, 872

Alexander, D. R., \& Fergusson, J. W. 1994, ApJ, 437, 879

Anders, E., \& Grevesse, N. 1989, Geochimica et Cosmochimica Acta 53, 197

Angulo, C., Arnould, M., Rayet, M., and the NACRE collaboration, 1999, Nuclear Physics A 656, 1, and WEb site http://pntpm.ulb.ac.be/Nacre/nacre.htm

Asplund, M., Carlsson, M., Garcia-Peres, A. E., \& Kiselman, D. 2000, Oxygen Abundances in Old Stars and Implications to Nucleosynthesis and Cosmology, in 24th meeting of the IAU, Joint Discussion 8, August 2000, Manchester, England

Asplund, M., Nordlund, A., Trampedach, R., \& Stein, R. F. 2000, A\&A, 359, 743

Baglin, A. 1972, A\&A, 19, 45

Balachandran, S. 1995, ApJ, 446, 203

Basu, S., \& Antia, H. M. 1995, MNRAS, 276, 1402

Basu, S. 1997, The Seismic Sun, in Sounding solar and stellar interiors, ed. J. Provost, \& F. X. Schmider (Kluwer Academic Publisher), IAU Symp., 181, 137

Bodmer, R., Bochsler, P., Geiss, J., et al. 1995, Space Sci. Rev., 72, 61

Boesgaard, A. M., \& Tripicco, M. 1986, ApJ, 302, 249

Böhm-Vitense, E. 1958, Z. Astrophys., 54, 114

Brun, S., Turck-Chièze, S., \& Zahn, J. P. 1999, ApJ, 525, 1032

Burgers, J. M. 1969, Flow equations for composite gases (Academic Press, New york and London)

Cayrel, R., Lebreton, Y., \& Morel, P. 1999, Survival of ${ }^{6} \mathrm{Li}$ and ${ }^{7} \mathrm{Li}$ in metal-poor stars, in Galaxy Evolution: Connecting the Distant Universe with the Local Fossil Record, ed. M. Spite, Astrophys. Space Sci. Lib., 265, Issue 1/4, 87

Chaboyer, B., Demarque, P., \& Guenther, D. B. 1999, ApJ, 525, L41

Chaboyer, B., Fenton, W. H., Nelan, J. E., et al. 2001, ApJ, 562, 521

Charbonnel, C., Vauclair, S., Maeder, A., et al. 1994, A\&A, 283, 155

Christensen-Dalsgaard, J., Däppen, W., Ajukov, S. V., et al. 1996, Science, 272, 1286

Cox, A. N., Guzik, J. A., \& Kidman, R. B. 1989, ApJ, 342, 1187

Cunha, K., \& Lambert, D. L. 1994, ApJ, 426, 170

Daflon, S., Cunha, K., Becker, S. R., \& Smith, V. V. 2001, ApJ, 552, 309

Eggleton, P. P., Faulkner, J., \& Flannery, B. P. 1973, A\&A, 23, 325

Gabriel, M. 1997, A\&A, 327, 771

Gautier, D., \& Morel, P. 1997, A\&A, 323, L9

Geiss, J., \& Reeves, H. 1972, A\&A, 18, 126

Grec, G., Turck-Chièze, S., Lazrek, M., et al. 1997, igolf results; to-day's view on the solar modes, in Sounding solar and stellar interiors, ed. J. Provost, \& F. X. Schmider (Kluwer Academic Publisher), IAU Symp., 181, 91

Grevesse, N., \& Noels, A. 1993, Cosmic Abundances of the Elements, in Origin and Evolution of the Elements, ed. N. Prantzos, E. Vangioni-Flam, \& M. Cassé (Cambridge University Press), 14

Heger, A., Langer, N., \& Woosley, S. E. 2000, ApJ, 528, 368

Hui-Bon-Hoa, A., \& Alecian, G. 1998, A\&A, 332, 224

Lebreton, Y., Perrin, M.-N., Cayrel, R., Baglin, A., \& Fernandes, J. 1999, A\&A, 350, 587 
Lebreton, Y., Fernandes, J., \& Lejeune, T. 2001, A\&A, 374, 540

Michaud, G., Tarasick, D., Charland, Y., \& Pelletier, C. 1983, ApJ, 269,239

Michaud, G., \& Proffitt, C. R. 1993, Particule Transport Process, in Inside the Stars, ed. A. Baglin, \& W. W. Weiss, IAU Coll., 137, ASP Conf. Ser., 40, 246

Mihalas, D. 1978, Stellar Atmospheres, 2d Ed. Freeman and Cie

Mihalas, D., \& Weibel-Mihalas, B. 1984, Foundations of Radiation Hydrodynamics (Oxford University Press), 461

Milne, E. A. 1930, Quart. J. of Math. (Oxford), 1, 1

Morel, P. 1997, A\&AS, 124, 597

Morel, P., Provost, J., \& Berthomieu, G. 1997, A\&A, 327, 349

Morel, P., \& Baglin, A. 1999, A\&A, 345, 156

Morel, P., Provost, J., Lebreton, Y., Thévenin, F., \& Berthomieu, G. 2000, A\&A, 363, 675

Paquette, C., Pelletier, C., Fontaine, G., \& Michaud, G. 1986, ApJS, 61,177

Proffit, C. R., \& Michaud, G. 1991, ApJ, 371, 584
Richard, O., Vauclair, S., Charbonnel, C., \& Dziembowsky, D. A. 1996, A\&A, 312, 1000

Richer, J., Michaud, G., \& Turcotte, S. 2000, ApJ, 529, 338

Rogers, F. J., Swenson, F. J., \& Iglesias, C. A. 1996, ApJ, 456, 902

Salaris, M., \& Weiss, A. 2001, A\&A, 376, 955

Schatzman, E. 1969, A\&A, 3, 331

Schatzman, E. 1977, A\&A, 56, 211

Schatzman, E., Zahn, J.-P., \& Morel, P. 2000, A\&A, 364, 876

Sills, A., \& Deliyannis, C. P. 2000, ApJ, 544, 944

Talon, S., \& Charbonnel, C. 1998, A\&A, 335, 959

Thomas, L. H. 1930, Quart. J. of Math. (Oxford), 1, 239

Thoul, A. A., Bahcall, J. N., \& Loeb, A. 1994, ApJ, 421, 828

Turck-Chièze, S., Basu, S., Brun, S., et al. 1997, Sol. Phys., 175, 247

Turcotte, S., Richer, J., Michaud, G., Iglesias, C. A., \& Rogers, F. J. 1998, ApJ, 504, 539

Turcotte, S., Richer, J., \& Michaud, G. 1998b, ApJ, 504, 559

Varenne, O., \& Monier, R. 1999, A\&A, 351, 247

Zahn, J.-P. 1992, A\&A, 265, 115 